\title{
Orbital Evolution of Planets around Intermediate-Mass Giants
}

\author{
M. Kunitomo*, M. Ikoma*, B. Sato*, Y. Katsuta ${ }^{\dagger}$ and S. Ida* \\ * Department of Earth and Planetary Sciences, Tokyo Institute of Technology, 2-12-1 Ookayama, \\ Meguro-ku, Tokyo 152-8551, Japan \\ ${ }^{\dagger}$ Department of Cosmosciences, Hokkaido University, Kita 10 Nishi 8, Kita-ku, Sapporo 060-0810, \\ Japan
}

\begin{abstract}
Around low- and intermediate-mass (1.5-3 $M_{\odot}$ ) red giants, no planets have been found inside $0.6 \mathrm{AU}$. Such a paucity is not seen in the case of $1 M_{\odot}$ main sequence stars. In this study, we examine the possibility that short-period planets were engulfed by their host star evolving off the main sequence. To do so, we have simulated the orbital evolution of planets, including the effects of stellar tide and mass loss, to determine the critical semimajor axis, $a_{\text {crit }}$, beyond which planets survive the RGB expansion of their host star. We have found that $a_{\text {crit }}$ changes drastically around $2 M_{\odot}$ : In the lower-mass range, $a_{\text {crit }}$ is more than $1 \mathrm{AU}$, while $a_{\text {crit }}$ is as small as about $0.2 \mathrm{AU}$ in the higher-mass range. Comparison with measured semimajor axes of known planets suggests that there is a lack of planets that only planet engulfment never accounts for in the higher-mass range. Whether the lack is real affects our understanding of planet formation. Therefore, increasing the number of planet samples around evolved intermediate-mass stars is quite meaningful to confirm robustness of the lack of planets.
\end{abstract}

Keywords: exoplanet, planetary system-formation and evolution, tide

PACS: $97.82 . \mathrm{Fs}, 96.15 . \mathrm{Bc}, 96.15 . \mathrm{Wx}$

\section{INTRODUCTION}

According to recent radial velocity surveys for GK giants, it appears that there is a lack of giant planets inside $0.6 \mathrm{AU}[18,13]$. Such a paucity is not seen around Sun-like stars which often harbor short-period planets such as hot Jupiters. One possibility is that such a property is primordial; namely, short-period giant planets (SPGPs) are not present around BA dwarfs originally. Another possibility is that SPGPs were removed during the evolution of their host star, which is to be explored in this paper.

A star evolves off the main sequence (MS) towards the red-giant branch (RGB), after exhaustion of hydrogen at its center. Then, once the central helium ignites, the star enters a next stable phase called the horizontal branch (HB). Most of the giants with detected planets are thought to be on their HB. Before reaching the HB, namely, in the RGB phase, the star expands substantially, becomes highly luminous, and loses substantial mass, which should affect orbits of surrounding planets.

Villaver \& Livio [23], following Sato et al. [18], examined what happened around evolving intermediate-mass stars to understand the observed lack of SPGPs around GK giants. They demonstrated that Jovian-mass planets initially orbiting at $<\sim 1 \mathrm{AU}$ around $2-3 M_{\odot}$ stars undergo orbital decay due to stellar tide, ending up being swallowed by their host star. However, they made no quantitative comparison with observation. 
The purpose of this paper is to examine whether planet engulfment by host stars is responsible for the lack of SPGPs, by making a quantitative comparison between the limits of existence of SPGPs predicted theoretically and suggested observationally. To this end, we derive a critical semimajor axis beyond which planets survive the host star's RGB phase, and investigate its sensitivity to stellar parameters.

\section{PHYSICAL MODEL}

We simulate the evolution of circular orbit of a planet during its host star's evolution. The effects of stellar tide and mass loss on planetary orbit are included. We neglect other competing processes such as the frictional and gravitational drag forces by stellar wind and change in the planet mass due to stellar-wind accretion and evaporation, which were evaluated to be negligible by Villaver \& Livio [23] and Duncan \& Lissauer [4]. Thus, we integrate

$$
\frac{1}{a} \frac{\mathrm{d} a}{\mathrm{~d} t}=-6 \frac{k}{T} \frac{M_{p}}{M_{\star}}\left(1+\frac{M_{p}}{M_{\star}}\right)\left(\frac{R_{\star}}{a}\right)^{8}-\frac{\dot{M}_{\star}}{M_{\star}},
$$

where $a$ is the semimajor axis, $t$ is time, $M_{p}$ is the planet's mass, $M_{\star}$ and $R_{\star}$ are the mass and radius of the host star, respectively, and $k$ is the apsidal motion constant, and $T$ is the eddy turnover timescale. The first term on the right-hand side represents the effect of stellar tide [7]. Since an RGB star is a slow rotator, we assume no stellar rotation, which means that the stellar tide always causes orbital decay of the planet. As for the parameters for stellar tide such as $k$ and $T$, we have followed Rasio et al. [15] and Villaver \& Livio [23].

The second term on the right-hand side of equation (1) represents orbital migration due to stellar mass loss $\left(\dot{M}_{\star}<0\right)$. We use the Reimers' parameterisation for stellar mass loss [16], namely, $\dot{M}_{\star}=-4 \times 10^{-13} \eta L_{\star} R_{\star} / M_{\star}$, where $\eta$ is the mass-loss parameter; $\dot{M}_{\star}$ is in $M_{\odot} \mathrm{yr}^{-1}$ and $M_{\star}, L_{\star}$, and $R_{\star}$ are in solar units.

We simulate stellar evolution directly with the code MESA v2258 [14] to calculate $M_{\star}, R_{\star}, \dot{M}_{\star}$, and parameters relevant to stellar tide as a function of time $t$. In this calculation in this paper, the effect of convective overshooting is not included (see Kunitomo et al. [10] for the results with overshooting).

\section{ORBITAL EVOLUTION AND SURVIVAL LIMIT}

Figure 1 shows the orbital evolution of planets around stars with masses of $2 M_{\odot}$ (upper panel) and $3 M_{\odot}$ (lower panel); the metallicity is solar $\left(Z_{\star}=0.02\right)$ in the calculations. The solid lines represent the evolution of the stellar radius $R_{\star}$. The orbit of a $1 M_{J}$ planet with different initial semimajor axes (dotted lines) has been integrated from the host-star's zero-age main sequence until the planet's semimajor axis falls below the stellar radius $\left(a<R_{\star}\right)$ or until the beginning of Asymptotic Giant Branch (AGB) thermal pulse.

Of special interest in this study is whether planets survive the RGB and HB phases of their host star. As seen in the upper panel of Fig. 1, the $2 M_{\odot}$ star expands up to $0.5 \mathrm{AU}$ at the RGB-tip and consequently swallows planets whose initial semimajor axes, $a_{i}$, are smaller than 1.1 AU. The planet that starts out at $1.2 \mathrm{AU}$ is pulled by the host star, but 

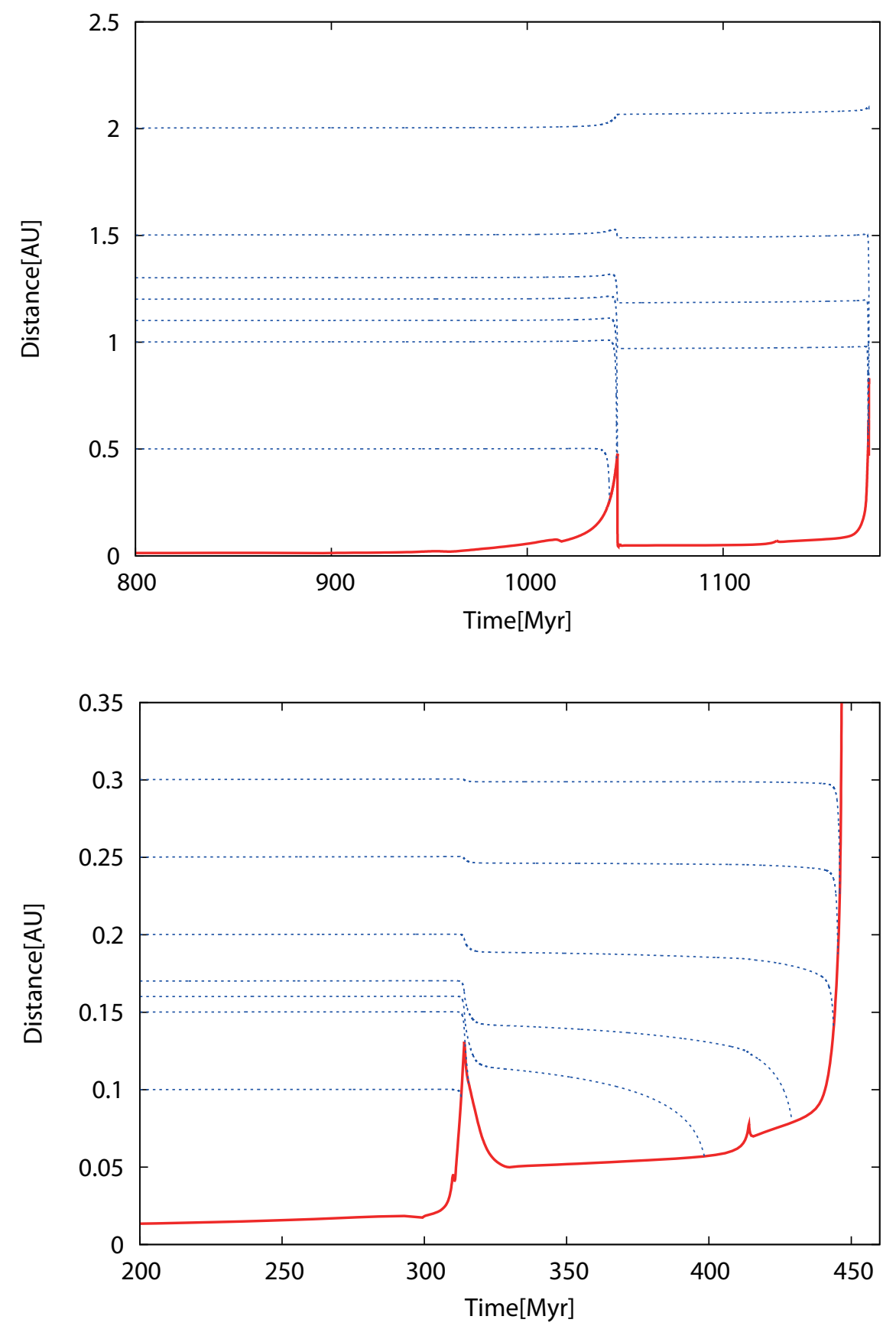

FIGURE 1. Evolution of semimajor axes of Jupiter-mass planets (dotted lines) and the radius of the solar-metallicity star (solid line) for $M_{\star}=2 M_{\odot}$ (upper panel) and $3 M_{\odot}$ (bottom panel).

it barely survives the RGB/HB phases. We define a critical initial semimajor axis, $a_{\text {crit }}$, below which planets end up being engulfed by their host star at some point on the RGB or HB. In the present case, $a_{\text {crit }}=1.2 \mathrm{AU}$. (Hereafter, we sometimes call the critical initial semimajor axis the survival limit.) As for the $3 M_{\odot}$ star (the lower panel of Fig. 1), the RGB-tip radius is as small as $0.13 \mathrm{AU}$. As a consequence, even a planet starting 


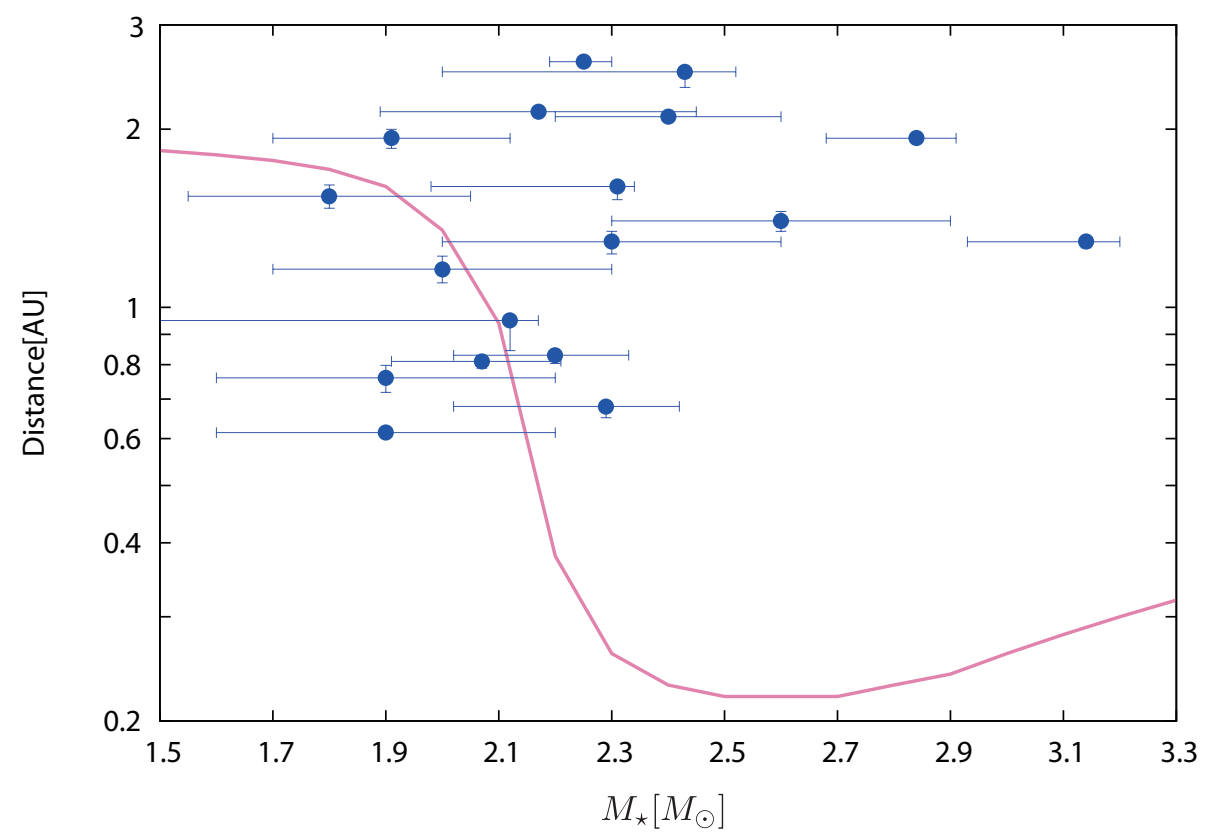

FIGURE 2. Comparison between the critical semimajor axis $a_{\text {crit }}$ (solid line) and measured semimajor axes of detected exoplanets (symbols with error bars). The theoretical curve is for stellar metallicity $Z=0.01$ and planetary mass $M_{p}=6 M_{J}$. In this calculation, the effect of overshooting is not included.

out at 0.20 AU can avoid engulfment during the RGB and HB phases. As seen in these panels, the survival limit is sensitive to the mass of host stars. While $a_{\text {crit }}=1.2 \mathrm{AU}$ for the $2 M_{\odot}$ star, $a_{\text {crit }}=0.20 \mathrm{AU}$ for the $3 M_{\odot}$ star.

\section{COMPARISON WITH OBSERVATION}

We compare measured semimajor axes of known planets around GK giants with the critical semimajor axis that we have derived in Fig. 2. Those measured stellar masses and planetary semimajor axes are taken from the literature [e.g., 22]. Two facts can be found in this figure: First, the observational errors being taken into account, it would be fair to say that all the known planets are outside the survival limit. Therefore, it is not denied that SPGPs $\left(a<a_{\text {crit }}\right)$ were swallowed by their host star on the RGB. Second, around giants of $M_{\star}>2.4 M_{\odot}$, planets exist far from $a_{\text {crit }}$. In other words, planet engulfment by host stars seems not to be the main reason for the lack of SPGPs.

Another possibility is that the lack of SPGPs is primordial - there may be any stellarmass-dependent processes that hinder formation of SPGPs around 2-3M $\odot$ stars. For example, Burkert \& Ida [1] proposed that a decreasing-with-stellar-mass lifetime of protoplanetary disks could become comparable with or shorter than the timescale of the type-II migration of giant planets. They then demonstrated that the observed period valley between the hot-Jupiter and cool-Jupiter classes is more pronounced for planets orbiting F stars $\left(1.2-1.5 M_{\odot}\right)$ than GK stars $\left(0.8-1.2 M_{\odot}\right)$. Applying the idea to high-mass stars $\left(1.5-3.0 M_{\odot}\right)$, Currie [2] demonstrated that SPGPs are rarely formed around high- 
mass stars.

Obviously we need more samples of giant planets and smaller planets orbiting highmass stars to confirm conclusions we have derived in this paper and to understand stellarmass-dependence of planet formation, which is also helpful in understanding the origins and diversity of planetary systems around solar-type stars. Therefore, further surveys for planets around giants are highly encouraged.

\section{ACKNOWLEDGMENTS}

We would like to express our gratitude to the following persons: B. Paxton and A. Dotter kindly helped us install and use the stellar-evolution code MESA and modified it upon our request. M. Fujimoto and T. Suda gave useful comments about stellar evolution. We had fruitful discussion on this study with Y. Hori and T. Nakamoto. This work is supported partly by Japan Society for the Promotion of Science (JSPS).

\section{REFERENCES}

1. Burkert, A., \& Ida, S. 2007, ApJ, 660, 845

2. Currie, T. 2009, ApJ, 694, L171

3. de Medeiros, J.R., et al. 2009, A\&A, 504, 617

4. Duncan, M. J., \& Lissauer, J. J. 1998, Icarus, 134, 303

5. Döllinger, M.P., Hatzes, A.P., Pasquini, L., Guenther, E.W., \& Hartmann, M. 2009, A\&A, 505, 1311

6. Hatzes, A.P., et al. 2006, A\&A, 457, 335

7. Hut, P. 1981, A\&A, 99, 126

8. Liu, Y.-J., Sato, B., Zhao, G., \& Ando, H. 2009, RAA, 9, L1

9. Lovis, C., \& Mayor, M. 2007, A\&A, 472, 657

10. Kunitomo, M., Ikoma, M., Sato, B., Katsuta, Y., \& Ida, S. 2011, ApJ, submitted

11. Niedzielski, A., et al. 2007, ApJ, 669, 1354

12. Niedzielski, A., Gozdziewski, K., Wolszczan, A., Konacki, M., Nowak, G., \& Zielinski, P. 2009, ApJ, 693, 276

13. Omiya, M., et al. 2009, PASJ, 61, 825

14. Paxton, B., Bildsten, L., Dotter, A., Herwig, F., Lesaffre, P., \& Timmes, F. 2010, arXiv:1009.1622

15. Rasio, F. A., Tout, C. A., Lubow, S. H., \& Livio, M. 1996, ApJ, 470, 1187

16. Reimers, D. 1975, in Problems in Stellar Atmospheres and Envelopes, ed. B. Bascheck,W. H. Kegel,

\& G. Traving (New York: Springer), 229

17. Sato, B., et al. 2007, ApJ, 661, 527

18. Sato, B., et al. 2008 , PASJ, 60,539

19. Sato, B., et al. 2008b, PASJ, 60, 1317

20. Sato, B., et al. 2010, PASJ, 62, 1063

21. Setiawan, J., et al. 2005, A\&A, 437, L31

22. Takeda, Y., Sato, B., \& Murata, D. 2008, PASJ, 60, 781

23. Villaver, E., \& Livio, M. 2009, ApJ, 705, L81 